\title{
Epithelial-mesenchymal transition-related gene expression as a new prognostic marker for neuroblastoma
}

\author{
MEGUMI NOZATO $^{1}$, SETSUKO KANEKO $^{1}$, AKIRA NAKAGAWARA $^{2}$ and HIROAKI KOMURO ${ }^{3}$ \\ ${ }^{1}$ Department of Pediatric Surgery, Faculty of Medicine, University of Tsukuba, Tsukuba; ${ }^{2}$ Chiba Cancer Center \\ Research Institute, Chiba; ${ }^{3}$ Department of Pediatric Surgery, Graduate School of Medicine, \\ University of Tokyo, Tokyo, Japan
}

Received August 14, 2012; Accepted October 9, 2012

DOI: 10.3892/ijo.2012.1684

\begin{abstract}
Neuroblastoma (NB) is a highly metastatic tumor in children. The epithelial-mesenchymal transition (EMT) is an important mechanism for both the initiation of tumor invasion and subsequent metastasis. This study investigated the role of EMT in the progression of NB. Using EMT assays on samples from 11 tumors, we identified 14 genes that were either differentially expressed between tumors of different stages or highly upregulated in NB. Quantitative RT-PCR of these genes was conducted in 96 NB tumors and their expression levels were compared between stages and between tumors with the presence and absence of MYCN amplification. The association of survival rate with differential gene expression was investigated. Expression of KRT19 was significantly decreased in stage 3 or $4 \mathrm{NB}$ as well as stage $4 \mathrm{~S} \mathrm{NB}$ compared with stage 1 or 2 NB. Expression levels of $K R T 19$ and $E R B B 3$ were significantly low, and expression levels of TWST1 and TCF3 were high in $M Y C N$-amplified NB. The patients with low expression of $K R T 19$ or ERBB3 showed significantly worse overall survival. Furthermore, the correlation between high invasive ability and low expression of KRT19 and ERBB3 was suggested in vitro using six NB cell lines. The authors conclude that downregulation of KRT19 is highly associated with tumor progression in NB and metastasis in localized primary NB and that low expression of $E R B B 3$ is also associated with progression of $\mathrm{NB}$.
\end{abstract}

\section{Introduction}

Neuroblastoma (NB) is one of the most common pediatric solid tumors, accounting for $15 \%$ of all pediatric cancer deaths. It originates from the sympathoadrenal lineage derived from the neural crest. The clinical behavior is markedly heteroge-

Correspondence to: Professor Hiroaki Komuro, Department of Pediatric Surgery, Graduate School of Medicine, University of Tokyo, 7-3-1 Hongo, Bunkyo-ku, Tokyo 113-8655, Japan

E-mail: komuroh-psu@h.u-tokyo.ac.jp

Key words: neuroblastoma, epithelial-mesenchymal transition, invasive ability, keratin19, ERBB3 (HER3) neous (1-3). Most tumors tend to grow aggressively and often have a fatal outcome, but some tumors are favorable and show spontaneous differentiation or regression. The stage of the tumor at diagnosis, the age of the patient and the presence or absence of $M Y C N$ amplification are the basic parameters used for risk stratification to determine the management and treatment of this disease. Recent progress in chemotherapy has dramatically increased the survival rates of many pediatric cancers; however, advanced stage NB with metastasis, especially those with genomic amplification of the $M Y C N$ oncogene, are frequently resistant to any therapy and the outcome for patients is still very poor (1-3). Therefore, it is important to know the mechanism of metastasis in NB in order to improve the treatment results.

The epithelial-mesenchymal transition (EMT) is a series of events during which epithelial cells lose many of their epithelial characteristics and take on properties typical of mesenchymal cells. EMT has an important role in the development of many tissues during embryogenesis and similar cell changes are recapitulated during pathological processes, such as fibrosis and cancer. Numerous observations support the idea that EMT has a central role in tumor progression and metastasis (4-7). Cancer cells acquire mesenchymal gene expression patterns and properties, resulting in reduced cell-cell adhesion and the activation of proteolysis and motility. These activities promote tumor invasion and metastasis. EMT is important in the progression of tumor cells acquiring a more invasive, metastatic capacity. In this study, we investigated the role of EMT in the progression of NB in terms of invasiveness and metastasis.

\section{Materials and methods}

Tumor samples. Ninety-six primary NBs were obtained from the Department of Pediatric Surgery, University of Tsukuba, and the Division of Biochemistry, Chiba Cancer Center Research Institute, Japan. Patients were aged between 0 months and 18 years at diagnosis (median 16 months). The clinical characteristics of the 96 NBs are shown in Table I.

Cell lines. Six NB cell lines (SK-N-AS, SK-N-DZ, SK-N-SH, GOTO, GANB and TGW) were used for invasion assays. SK-N-SH, SK-N-DZ and SK-N-AS were kindly provided by Toru Sugimoto, Kyoto Prefectural Medical University. TGW and GANB were provided by Chiba Cancer Center. GOTO was 
Table I. Tumor stages and MYCN amplification of 96 neuroblastomas.

Stage 1, 2 Stage 4S Stage 3 Stage 4 Total

\begin{tabular}{lrrrrr}
\hline MYCN & & & & & \\
Unamplified & 22 & 4 & 10 & 15 & 51 \\
Amplified & 2 & 2 & 11 & 30 & 45 \\
Total & 24 & 6 & 21 & 45 & 96 \\
\hline
\end{tabular}

purchased from American Type Culture Collection (Manassas, VA, USA). These were maintained in Daigo's medium supplemented with $10 \%$ fetal bovine serum (BioWest, Nuaille, France) at $37^{\circ} \mathrm{C}$ in a humidified $5 \% \mathrm{CO}_{2}$ atmosphere.

RNA extraction and cDNA transcription. Total-RNA was prepared from frozen tumor tissue by the guanidine isothiocyanate-phenol method using Isogen (Wako Junyaku Kogyo, Tokyo, Japan) according to the manufacturer's instructions. One microgram of each RNA was reverse transcribed to cDNA with random hexamer primers and transcriptor reverse transcriptase using the Transcriptor First Strand cDNA Synthesis Kit (Roche, USA).

EMT assay. To examine the expression levels of the EMT-related genes, we used an $\mathrm{RT}^{2}$ Profiler PCR Array for human EMT (SA Biosciences) consisting of quantitative RT-PCR of 84 EMT-related genes. This array coated 96-well microtiter plates and was performed using an ABI Prism 7700 Sequence Detection System (Applied Biosystems, Foster City, CA, USA) according to the following program: $95^{\circ} \mathrm{C}$ for $10 \mathrm{~min}, 43$ cycles at $95^{\circ} \mathrm{C}$ for $15 \mathrm{sec}$ and then $60^{\circ} \mathrm{C}$ for $1 \mathrm{~min}$.

Real-time quantitative RT-PCR. The expression levels of cardesmon $1(C A L D 1)$, epidermal growth factor $(E G F R)$, desmoplakin $(D S P)$, secreted protein acidic and rich in cysteine (SPARC), zinc finger E-box-binding homeobox 1 (ZEBI), zinc finger E-box-binding homeobox 2 (ZEB2), fibronectin 1 (FNI), vimentin (VIM), keratin 19 (KRT19), erythroblastic leukemia viral oncogene homolog (ERBB3), regulator of G-protein signaling 2 (RGS2), transcription factor 3 (TCF3) and TWISTI were measured by the ABI Prism 7700 Sequence Detection System (Applied Biosystems) using Universal ProbeLibrary (UPL)-based real-time quantitative RT-PCR (Roche Diagnostics). UPL is based on only 165 short hydrolysis probes of just 8-9 nucleotides, each of which is labeled at the 5 ' end with FAM and at the 3 ' end with a dark quencher dye. Human $A C T N B$ ( $\beta$-actin) was used as an internal control gene. The specific primers used are shown in Table II. The UPL probes used were nos. 52, 69, 78, 7, 77, 3, 68, 13, 33, 71, 37, 61, 35, 6 and 64 in UPL for CALD1, EGFR, DSP, SNAIL2, SPARC, ZEB1, ZEB2, VIM, FN1, KRT19, ERBB3, RGS2, TCF3, TWIST1 and human $A C T N B$, respectively. Each experiment was carried out with each sample in triplicate and repeated twice. The thermal cycling conditions were as follows: $50^{\circ} \mathrm{C}$ for $2 \mathrm{~min}, 95^{\circ} \mathrm{C}$ for $10 \mathrm{~min}, 40$ cycles at $95^{\circ} \mathrm{C}$ for $15 \mathrm{sec}$ and then $60^{\circ} \mathrm{C}$ for $1 \mathrm{~min}$. Data from real-time PCR were calculated using the $\Delta \Delta \mathrm{C}_{\mathrm{t}}$ method as previously described (8).

Matrigel invasion assay. The invasive ability of NB cell lines was measured using BD Falcon cell culture inserts with an 8- $\mu \mathrm{m}$ pore size PET membrane and 24-well BD BioCoat Matrigel Invasion Chambers (BD Biosciences, Bedford, MA, USA) according to the manufacturer's instructions. NB cell suspensions were adjusted to $1.0 \times 10^{5}$ cells per well on Matrigel invasion chamber plates and non-matrigel coat invasion chamber (control inserts) and cultured in routine medium in the absence or presence of FBS. After incubation at $37^{\circ} \mathrm{C}$ under $5 \% \mathrm{CO}_{2}$ for $72 \mathrm{~h}$, the cells that had invaded the chamber and migrated to the lower surface were stained with DiffQuik (Sysmex, Kobe, Japan) and manually counted under a microscope. The invading cells were stained and counted in

Table II. Sequences of the primers used for PCR.

\begin{tabular}{lll}
\hline Gene & \multicolumn{1}{c}{ Forward primer } & \multicolumn{1}{c}{ Reverse primer } \\
\hline$C A L D 1$ & 5'-GAGCGTCGCAGAGAACTTAGA-3' & 5'-TCCTCTGGTAGGCGATTCTTT-3' \\
$E G F R$ & 5'-GCCTTGACTGAGGACAGCA-3' & 5'-TTTGGGAACGGACTGGTTTA-3' \\
$D S P$ & 5'-CTTTGCGCCAATTCAATTAAG-3' & 5'-CCAGTCCTGAGGTGTATGAGG-3' \\
SNAIL2 & 5'-TGGTTGCTTCAAGGACACAT-3' & 5'-GTTGCAGTGAGGGCAAGAA-3' \\
SPARC & 5'-GTGCAGAGGAAACCGAAGAG-3' & 5'-TGTTTGCAGTGGTGGTTCTG-3' \\
$Z E B$ I 2 & 5'-GGGAGGAGCAGTGAAAGAGA-3' & 5'-TTTCTTGCCCTTCCTTTCTG-3' \\
$V I M$ & 5'-AAGCCAGGGACAGATCAGC-3' & 5'-CCACACTCTGTGCATTTGAACT-3' \\
$F N 1$ & 5'-TACAGGAAGCTGCTGGAAGG-3' & 5'-ACCAGAGGGAGTGAATCCAG-3' \\
KRT19 & 5'-GGAAAGTGTCCCTATCTCTGATACC-3' & 5'-AATGTTGGTGAATCGCAGGT-3' \\
$E R B B 3$ & 5'-GCCACTACTACACGACCATCC-3' & 5'-CAAACTTGGTTCGGAAGTCAT-3' \\
$R G S 2$ & 5'-CTGATCACCGGCCTCAAT-3' & 5'-GGAAGACATTGAGCTTCTCTGG-3' \\
$T C F 3$ & 5'-GAAAAGGAAGCTCCAAAAGAGA-3' & 5'-TTCTGGGCAGTTGTAAAGCA-3' \\
$T W I S T 1$ & 5'-CTCGGTCATCCTGAACTTGG-3' & 5'-TCTCCAACCACACCTGACAC-3' \\
$A C T N B$ & 5'-AAGGCATCACTATGGACTTTCTCT-3' & 5'-GCCAGTTTGATCCCAGTATTTT-3' \\
& 5'-CCAACCGCGAGAAGATGA-3' & 5'-CCAGAGGCGTACAGGGATAG-3'
\end{tabular}


Table III. Characteristics of 11 neuroblastomas used in EMT assay.

Stage 1,2 Stage 4S Stage 3,4 Total

\begin{tabular}{llllr}
\hline MYCN & & & & \\
Unamplified & 1 & 1 & 1 & 3 \\
Amplified & 3 & 2 & 3 & 8 \\
Total & 4 & 3 & 4 & 11 \\
\hline
\end{tabular}

5 random fields at x100 magnification. The mean number of counted cells was defined as the invasive ability. Each experiment was repeated 3 times.

Statistical analysis. Survival analysis was performed according to the Kaplan-Meier method and the log-rank test. Relative mRNA expression levels were expressed as the mean $\pm \mathrm{SD}$. Student's or Welch's t-tests were used to assess the significance of differences between the groups. A p-value of $<0.01$ was considered statistically significant. This study was approved by the institutional ethics committee for human genome research of the University of Tsukuba (no. 211).

\section{Results}

Analysis of EMT-related gene expression in $11 \mathrm{NB}$ tumors using EMT assay. Eleven NB tumors in various stages (Table III) were analyzed by EMT multiple gene profiling microarray. The
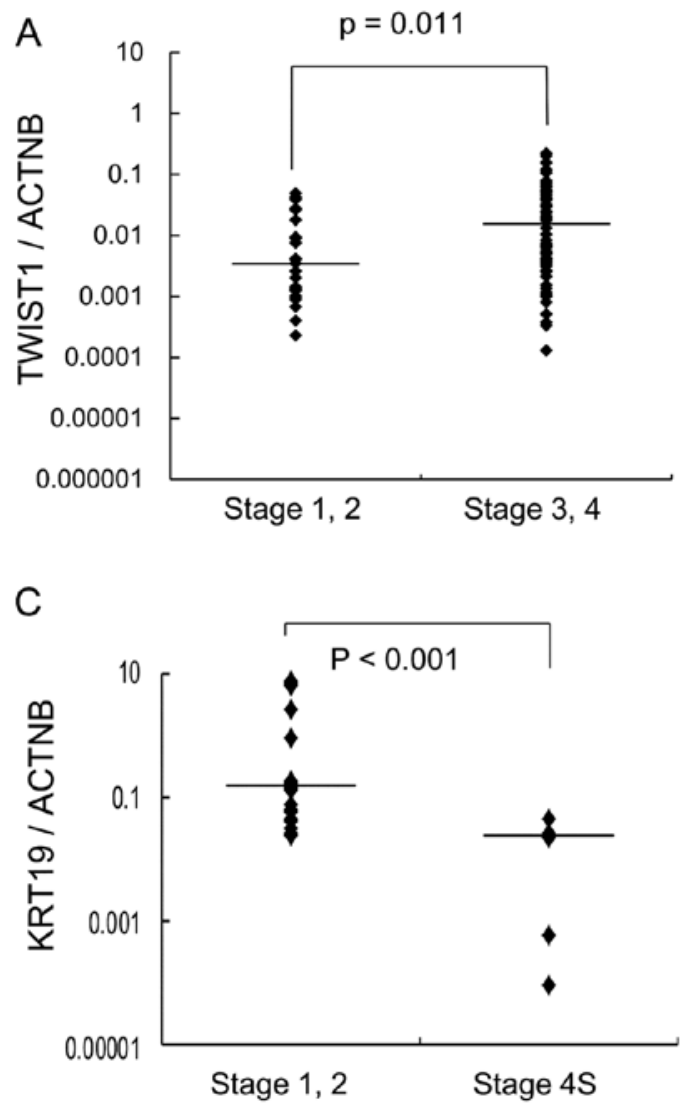

expressions of 84 EMT-related genes were compared among the 11 tumors using the EMT assay. Seven genes (CALDI, EGFR, DSP, SNAIL2, SPARC, ZEBI and ZEB2) were found to be differentially expressed between NBs with low stages (stages 1 or 2) and those with high stages (stages 3 or 4 ). Five genes (KRT19, ERBB3, RGS2, TCF3 and TWISTI) were found to be differentially expressed between $M Y C N$-amplified and $M Y C N$-non-amplified tumors. These genes and two others highly expressed in NB tumors (VIM and FNI) were further analyzed in 96 tumors using quantitative PCR.

Correlation of EMT-related gene expression between low and high tumor stages. The expression levels of ERBB3, RGS2, TCF3, CALD1, EGFR, DSP, SNAIL2, SPARC, ZEB1, ZEB2, $V I M$ and $F N I$ did not show any significant differences between low- and high-stage NB. In contrast, low expression of KRT19 was significantly associated with high stages of NB (Fig. 1B). TWIST1 was found to be highly expressed in stage 3 or $4 \mathrm{NB}$ $(\mathrm{p}=0.011)($ Fig. 1A).

Correlation of EMT-related gene expression with metastasis in localized primary tumors. Expression of these EMT-related genes was compared between stage 1 or 2 localized NB and stage 4S NB. Expression of KRT19 was significantly lower in stage $4 \mathrm{~S} \mathrm{NB}$, which develops metastasis in localized primary NB (Fig. 1C).

Correlation of EMT-related gene expression with MYCN amplification. Expression of these EMT-related genes was compared between NB with and without $M Y C N$ amplification.

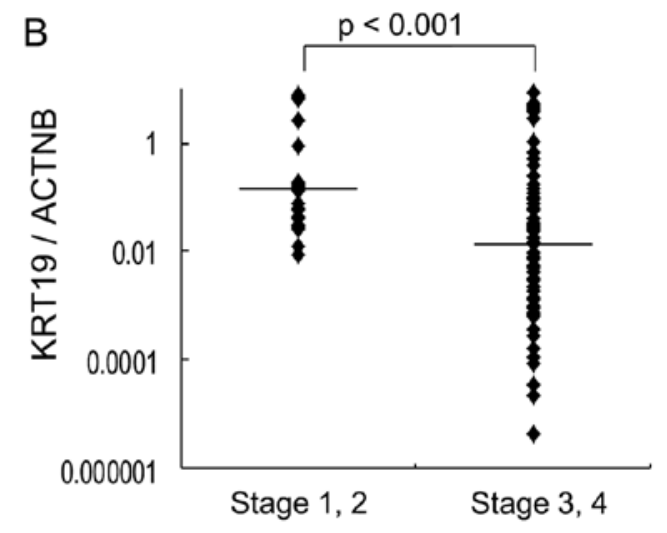

Figure 1. (A and B) Differential expression of EMT-related genes between lowstage NB (stage 1 or 2) and high-stage NB (stage 3 or 4). (A) TWIST1 was more highly expressed in high-stage NB $(\mathrm{p}=0.011)$. (B) Significantly low expression of KRT19 was associated with high stages of NB. (C) Differential expression of KRT19 between localized stage 1 or $2 \mathrm{NB}$ and metastatic localized stage 4S NB. KRT19 was downregulated in metastatic stage 4S NB compared with localized stage 1 or $2 \mathrm{NB}$. 
A

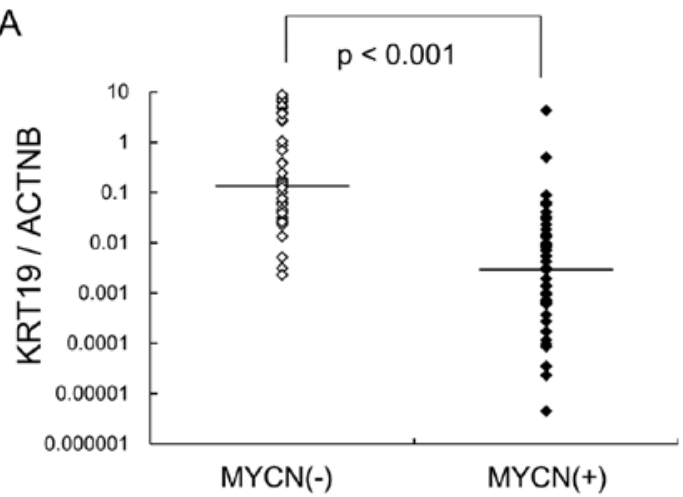

C

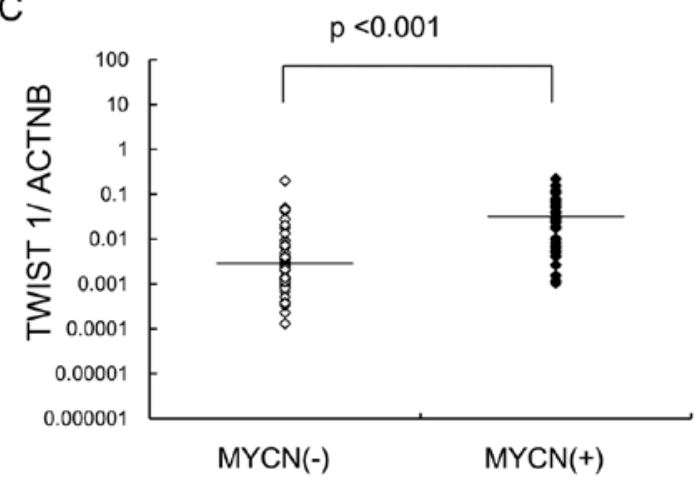

B

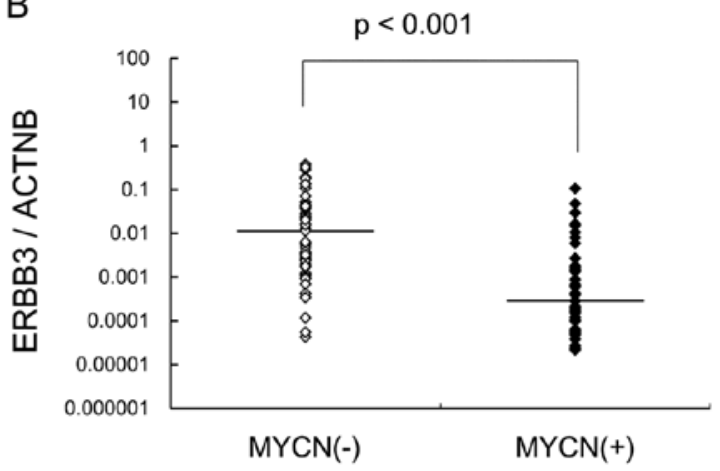

$\mathrm{D}$

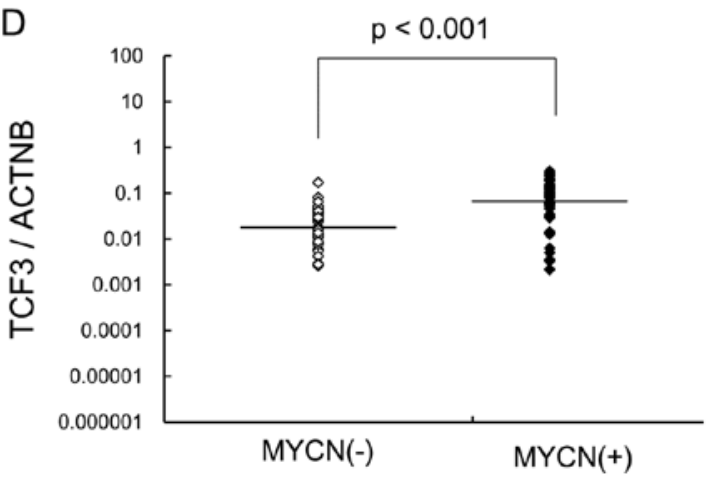

Figure 2. Differential expression of EMT-related genes between $M Y C N$-amplified and $M Y C N$-unamplified NB. (A and B) Expression of $K R T 19$ and $E R B B 3$ was significantly decreased in $M Y C N$-amplified NB, (C and D) while expression of $T W I S T 1$ and $T C F 3$ was significantly increased in $M Y C N$-amplified NB. MYCN(+), $M Y C N$ amplification; $M Y C N(-), M Y C N$ non-amplification.
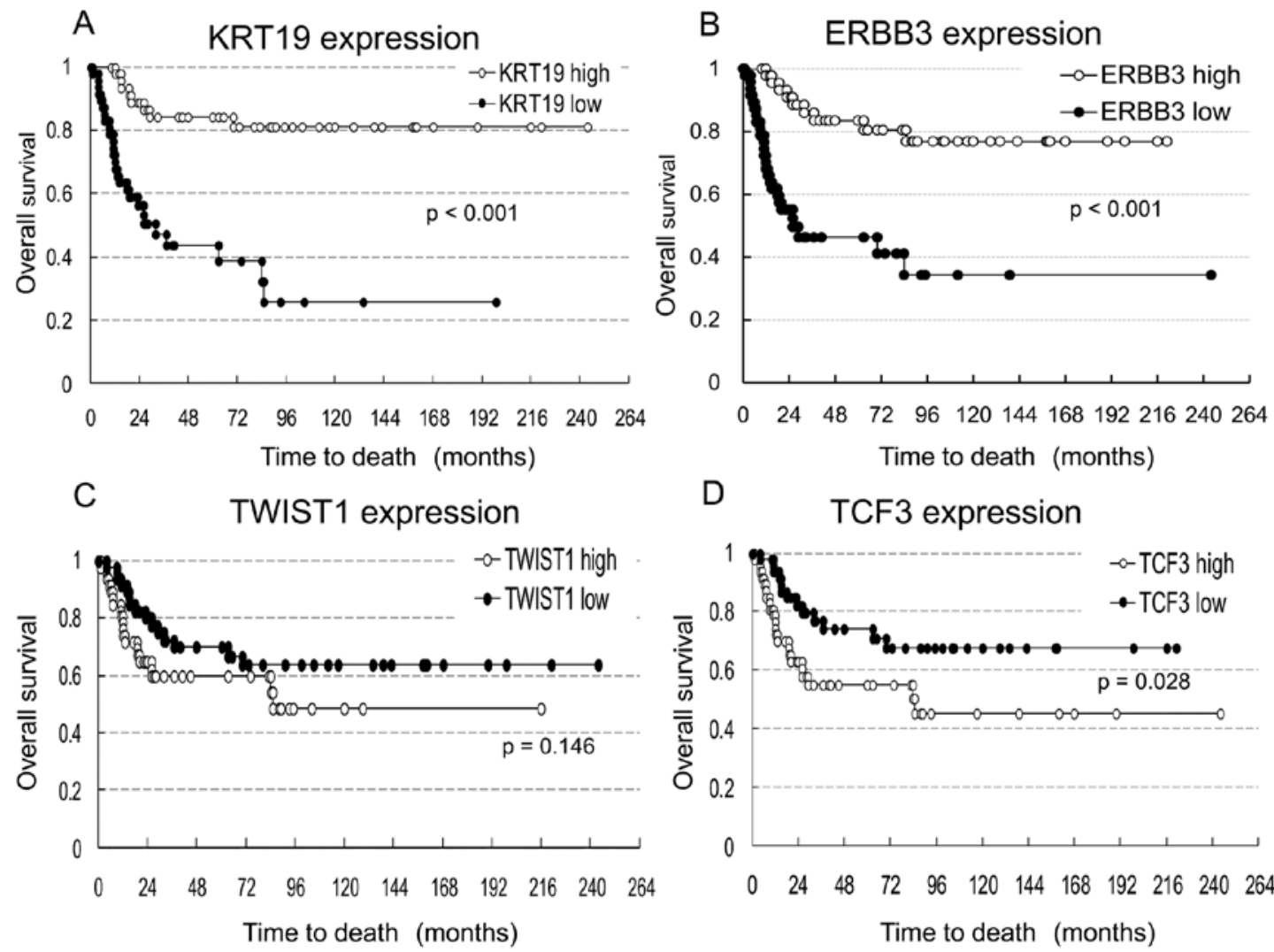

Figure 3. Kaplan-Meier survival analysis of 94 NB patients, stratified by their status of KRT19, ERBB3, TWIST1 and TCF3 gene expression. (A and B) The patients with low expression of KRT19 or ERBB3 in tumor tissues had significantly inferior survival compared with those with high expression. (C and D) No significant difference was observed between patients with high and low expression of TWIST1 and TCF3 genes. 
A

\begin{tabular}{|c|c|c|}
\hline Cell line & Invasionability (\%) & MYCNamplification \\
\hline SK-N-DZ & 4.12 & + \\
\hline SK-N-SH & 6.75 & - \\
\hline GANB & 24.8 & + \\
\hline TGW & 45.5 & + \\
\hline SK-N-AS & 50.86 & - \\
\hline GOTO & 62.14 & + \\
\hline
\end{tabular}

B

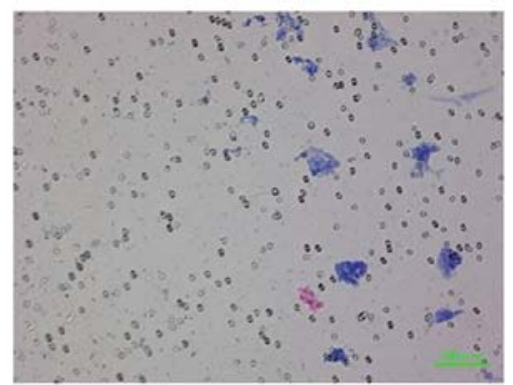

C

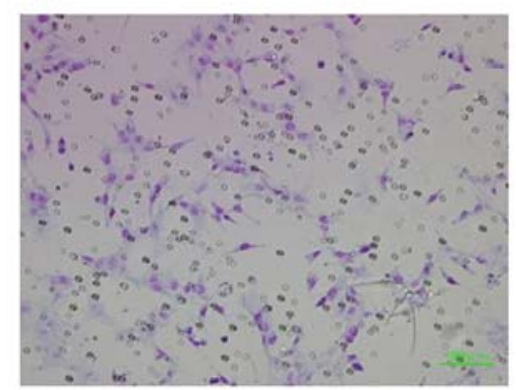

D

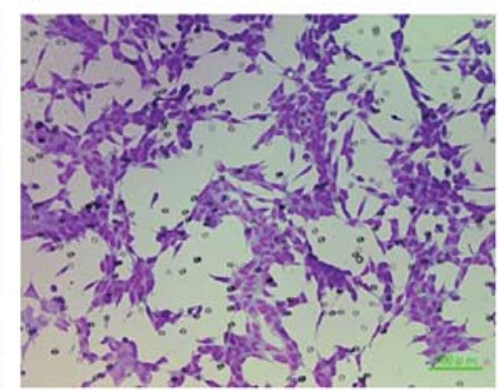

Figure 4. Results of Matrigel invasion assay in 6 NB cell lines. (A) Two cell lines (SK-N-DZ and SK-N-SH) showed low invasive abilities, while the other four cell lines (GANB, TGW, SK-N-AS and GOTO) showed high invasive abilities. (B) SK-N-DZ, (C) SK-N-AS and (D) GOTO cells are shown to be capable of migrating through the matrigel.

Expression of KRT19 and ERBB3 was significantly decreased in NB with MYCN amplification, while TCF3 and TWIST1 expression were increased (Fig. 2). $M Y C N$-amplified NB showed significantly lower expression of KRT19 and ERBB3 compared with $M Y C N$-unamplified NB.

Overall survival rates for tumors with VIM, FN1, KRT19, ERBB3, TCF3 and TWIST1 gene misregulation. Survival analysis was conducted in 94 NB tumors excluding 2 in which NB was not the cause of death. These NBs were divided into two groups: high expressers (47 NBs) and low expressers (47 NBs) of 6 genes (VIM, FN1, KRT19, ERBB3, TCF3 and TWIST1). The median of log-transformed mRNA expression level was used as the cut-off value. Kaplan-Meier survival curves were compared for each gene between tumors with high and low expression (Fig. 3). The graph shows a trend toward increased survival for NB patients with increased KRT19 or ERBB3 expression. Expression levels of the other genes (VIM, FN1, $T C F 3$ and TWIST1) were not associated with patient survival.

The correlation of low KRT19 and ERBB3 expression with invasive ability in $\mathrm{NB}$ cell lines. A Matrigel invasion assay demonstrated that two cell lines (SK-N-SH and SK-N-DZ) showed significantly reduced invasive ability (6.75 and $4.12 \%$, respectively) while the 4 other cell lines (GANB, TGW, SK-N-AS and GOTO) showed high invasive abilities (24.8, $45.5,50.86$ and $62.1 \%$, respectively) (Fig. 4). The correlation of KRT19 and ERBB3 expression with invasive abilities was investigated in the cell lines. The decreased expression of $K R T 19$ or ERBB3 was highly correlated with invasiveness in NB cell lines (Fig. 5A and B). SK-N-DZ, with high expression
A

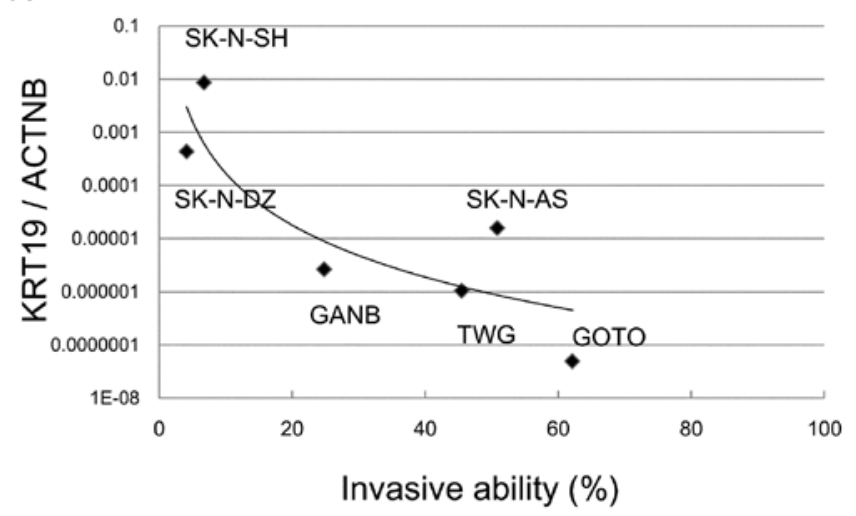

B

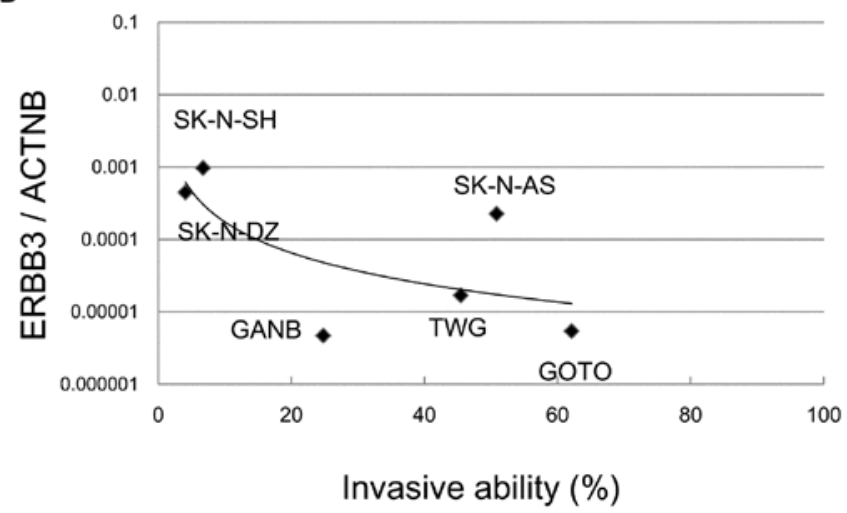

Figure 5. Effects of ERBB3 and KRT19 mRNA expression on in vitro tumor cell invasiveness. Note the markedly decreased invasive abilities in the cell lines with high expression of (A) KRT19 and (B) ERBB3 in comparison to those with low expression. 
of $K R T 19$ and ERBB3 and MYCN amplification, had low invasive ability; while SK-N-AS, with low expression of KRT19 and $M Y C N$ non-amplification, showed high invasive ability.

\section{Discussion}

In this study, four EMT-related genes (KRT19, ERBB3, $T W I S T 1$ and $T C F 3$ ) were found to be differentially expressed. Expression of $K R T 19$ was significantly decreased in high-stage NB compared to low-stage NB (Fig. 1B). Downregulation of KRT19 gene expression was highly associated with tumor progression in NB. Furthermore, expression of KRT19 was markedly decreased in NB with $M Y C N$ amplification.Decreased expression of KRT19 was found to be significantly associated with poor prognosis (Fig. 3). Interestingly, expression of KRT19 was significantly decreased in metastatic favorable stage $4 \mathrm{~S}$ NB compared to localized favorable stage 1 or 2 NB (Fig. 1C). These findings show that decreased expression of KRT19 is strongly associated with the promotion of metastasis in favorable NB. Keratin is an epithelial marker, and downregulation of keratins is associated with EMT. Dysregulation of keratin expression has long been recognized as a feature of epithelial tumor progression (9). A recent report also demonstrated that expression of KRT19 mRNA was significantly lower in tumors from patients that have died from NB compared with patients with no evidence of disease, and that low methylation of KRT19 was associated with a favorable outcome (10). Supporting this, our results demonstrated that low expression of KRT19 was significantly associated with high tumor stages, $M Y C N$ amplification and an unfavorable outcome in NB.

TWIST1 is a key regulator of embryogenesis and is also known to be an EMT inducer. TWIST1 belongs to the basic helix-loop-helix (bHLH) transcription factor family and promotes EMT by repressing the expression of E-cadherin, which leads to disassembly of adherens junctions and increased migratory potential (11). The link between TWISTl expression and metastasis is clear and well established (11,12). TWIST1 is known to be overexpressed in $M Y C N$-amplified NB tumors and cell lines and is responsible for the inhibition of the ARF/p53 pathway involved in the MYC-dependent apoptotic response. The cooperation of TWISTI and MYCN is thought to cause cell transformation and malignant outgrowth $(13,14)$. In this study, TWIST1 was highly expressed in $M Y C N$-amplified NB as well as in high-stage NB. However, the survival rates between patients with low and high expression of TWIST1 were not significantly different $(\mathrm{p}=0.146)$, so its utility as a mesenchymal marker may be limited.

TCF3 (E12/E47) is a basic bHLH transcription factor. A previous study implicated TCF3 as a repressor of E-cadherin promoter activity and demonstrated its involvement in the acquisition and maintenance of the mesenchymal phenotype (15). In this study, high expression of TCF3 was associated with $M Y C N$ amplification in NB. Survival rates were not significantly different between patients with high and low expression of $T C F 3$.

$E R B B 3$ is a member of the epidermal growth factor receptor (EGFR) family, which is composed of EGFR, ERBB2 (HER2), $E R B B 3$ (HER3) and ERBB4 (HER4). Although ERBB3 lacks an active tyrosine kinase domain, it can heterodimerize with other $E R B B$ receptors. Heterodimerization leads to the activation of pathways which lead to cell proliferation or differentiation. The role of EGFR in the proliferation of NB, and the utility of its inhibitors in the treatment of $\mathrm{NB}$, have all been well documented; however, the data remain somewhat contradictory $(16,17)$, as other reports have demonstrated that exposure to EGF can induce apoptosis in NB through the ERBB2 and ERBB3 receptors (18-20). Richards et al reported that non-EGFR ERBB family members (ERBB2, ERBB3 and $E R B B 4)$ contributed to NB growth and survival, and that pan-ERBB inhibition, rather than an EGFR specific inhibitor, represents a potential therapeutic target (21). These findings suggest that $E R B B 2, E R B B 3$ and $E R B B 4$ play a significant role in tumor progression of $\mathrm{NB}$, but Gambini et al reported that expression of $E R B B 2$ was not related to tumor progression of NB (22). Although a recent immunohistological study suggested the significance of EGFR family expression as a prognostic factor in NB, showing that EGFR and HER 2 expression is found in favorable NB and high expression of HER4 is found in metastatic NB, the role of HER family members in NB remains interrelated and complex (23). In our study, decreased expression of $E R B B 3$ was also correlated with $M Y C N$-amplified NB and poor survival rate. Several lines of evidence that provide support for the pivotal role of ERBB3 in human carcinogenesis have emerged in recent years (24). High expression of ERBB3 in certain human cancers led early to the suggestion that it could be a therapeutic target (25-28), but in some cancer cells the mesenchymal phenotype was found to lose $E R B B 3$ expression and show resistance to EGFR inhibitors. Epithelial phenotype, however, maintained ERBB3 expression (29,30). The EMT might decrease the cellular dependency upon EGF signaling by kinase switching; mesenchymal cells might acquire alternative survival signals, thus becoming resistant to EGFR inhibitors (30). Downregulation of ERBB3 in NB might suggest similar kinase switching during the EMT followed by tumor survival with the loss of EGF dependency.

Next, we investigated the invasive abilities of six NB cell lines using a Matrigel invasion assay to confirm the association of tumor invasiveness with expression of KRT19 and ERBB3. While SK-N-DZ and SK-N-SH cell lines had a low invasive ability (4.12 and 6.75\%, respectively), the other cell lines showed a high invasive ability (24.8-62.14\%) (Fig. 4). Both cell lines with a low invasive ability had low expression of KRT19 and ERBB3 compared with the other cell lines (Fig. 5A and B). Interestingly, SK-N-DZ showed a low invasive ability as expected from high expression levels of KRT19 and ERBB3, although its $M Y C N$ amplification should give it a high invasive ability. Thus, although $M Y C N$ gene amplification is the most powerful prognostic factor in NB, the expression levels of KRT19 or ERBB3 might become another promising prognostic marker.

\section{Acknowledgements}

This study was supported by a Grant-in-Aid for Challenging Exploratory Research from the Japan Society for the Promotion of Science (JSPS) Grant 22659317 (to HK).

\section{References}

1. Brodeur GM: Neuroblastoma: biological insights into a clinical enigma. Nat Rev Cancer 3: 203-216, 2003.

2. Maris JM: Recent advances in neuroblastoma. N Engl J Med 362: 2202-2211, 2010 
3. Maris JM: The biologic basis for neuroblastoma heterogeneity and risk stratification. Curr Opin Pediatr 17: 7-13, 2005.

4. Thiery JP and Sleeman JP: Complex networks orchestrate epithelial-mesenchymal transitions. Nat Rev Mol Cell Biol 7: 131-142, 2006.

5. Yang $\mathrm{J}$ and Weinberg RA: Epithelial-mesenchymal transition: at the crossroads of development and tumor metastasis. Dev Cell 14: 818-829, 2008.

6. Lee JM, Dedhar S, Kalluri R and Thompson EW: The epithelial-mesenchymal transition: new insights in signaling, development, and disease. J Cell Biol 172: 973-981, 2006.

7. Thiery JP: Epithelial-mesenchymal transitions in tumour progression. Nat Rev Cancer 2: 442-454, 2002.

8. Livak KJ and Schmittgen TD: Analysis of relative gene expression data using real-time quantitative PCR and the $2^{-\Delta \Delta C}$ method. Methods 25: 402-408, 2001.

9. Moll R, Franke WW, Schiller DL, Geiger B and Krepler R: The catalog of human cytokeratins: patterns of expression in normal epithelia, tumors and cultured cells. Cell 31: 11-24, 1982.

10. Carén H, Djos A, Nethander M, Sjöberg RM, Kogner P, Enström C, Nilsson S and Martinsson T: Identification of epigenetically regulated genes that predict patient outcome in neuroblastoma. BMC Cancer 11: 66, 2011.

11. Yang J, Mani SA, Donaher JL, Ramaswamy S, Itzykson RA, Come C, Savagner P, Gitelman I, Richardson A and Weinberg RA: Twist, a master regulator of morphogenesis, plays an essential role in tumor metastasis. Cell 117: 927-939, 2004.

12. Karreth F and Tuveson DA: Twist induces an epithelial-mesenchymal transition to facilitate tumor metastasis. Cancer Biol Ther 3: 1058-1059, 2004.

13. Valsesia-Wittmann S, Magdeleine M, Dupasquier S, Garin E Jallas AC, Combaret V, Krause A, Leissner P and Puisieux A: Oncogenic cooperation between H-Twist and N-Myc overrides failsafe programs in cancer cells. Cancer Cell 6: 625-630, 2004.

14. Puisieux A, Valsesia-Wittmann S and Ansieau S: A twist for survival and cancer progression. Br J Cancer 94: 13-17, 2006.

15. Perez-Moreno MA, Locascio A, Rodrigo I, Dhondt G, Portillo F Nieto MA and Cano A: A new role for E12/E47 in the repression of E-cadherin expression and epithelial-mesenchymal transitions. J Biol Chem 276: 27424-27431, 2001.

16. Ho R, Minturn JE, Hishiki T, Zhao H, Wang Q, Cnaan A, Maris J, Evans AE and Brodeur GM: Proliferation of human neuroblastomas mediated by the epidermal growth factor receptor. Cancer Res 65: 9868-9875, 2005.

17. Tamura S, Hosoi H, Kuwahara Y, Kikuchi K, Otabe O, Izumi M, Tsuchiya K, Iehara T, Gotoh T and Sugimoto T: Induction of apoptosis by an inhibitor of EGFR in neuroblastoma cells. Biochem Biophys Res Commun 358: 226-232, 2007.
18. Chiu B, Mirkin B and Madonna MB: Epidermal growth factor can induce apoptosis in neuroblastoma. J Pediatr Surg 42: 482-488, 2007

19. Chiu B, Mirkin B and Madonna MB: Mitogenic and apoptotic actions of epidermal growth factor on neuroblastoma cells are concentration-dependent. J Surg Res 135: 209-212, 2006.

20. Chiu B, Mirkin B and Madonna MB: Novel action of epidermal growth factor on caspase 3 and its potential as a chemotherapeutic adjunct for neuroblastoma. J Pediatr Surg 42: 1389-1395, 2007.

21. Richards KN, Zweidler-McKay PA, Van Roy N, Speleman F, Trevino J, Zage PE and Hughes DP: Signaling of ERBB receptor tyrosine kinases promotes neuroblastoma growth in vitro and in vivo. Cancer 116: 3233-3243, 2010.

22. Gambini C, Sementa AR, Boni L, Marino CE, Croce M Negri F, Pistoia V, Ferrini S and Corrias MV: Expression of HER2/neu is uncommon in human neuroblastic tumors and is unrelated to tumor progression. Cancer Immunol Immunother 52: 116-120, 2003.

23. Izycka-Swieszewska E, Wozniak A, Drozynska E, Kot J Grajkowska W, Klepacka T, Perek D, Koltan S, Bien E and Limon J: Expression and significance of HER family receptors in neuroblastic tumors. Clin Exp Metastasis 28: 271-282, 2011.

24. Sithanandam G and Anderson LM: The ERBB3 receptor in cancer and cancer gene therapy. Cancer Gene Therapy 15: 413-448, 2008

25. Gullick WJ: The c-erbB3/HER3 receptor in human cancer Cancer Surv 27: 339-349, 1996.

26. Travis A, Pinder SE, Robertson JF, Bell JA, Wencyk P, Gullick WJ, Nicholson RI, Poller DN, Blamey RW, Elston CW and Ellis IO: C-erbB-3 in human breast carcinoma: expression and relation to prognosis and established prognostic indicators. Br J Cancer 74: 229-233, 1996.

27. Sergina NV, Rausch M, Wang D, Blair J, Hann B, Shokat KM and Moasser MM: Escape from HER-family tyrosine kinase inhibitor therapy by the kinase-inactive HER3. Nature 445: 437-441, 2007

28. Baselga $\mathbf{J}$ and Swain SM: Novel anticancer targets: revisiting ERBB2 and discovering ERBB3. Nat Rev Cancer 9: 463-475, 2009.

29. Fuchs BC, Fujii T, Dorfman JD, Goodwin JM, Zhu AX, Lanuti $M$ and Tanabe KK: Epithelial-to-mesenchymal transition and integrin-linked kinase mediate sensitivity to epidermal growth factor receptor inhibition in human hepatoma cells. Cancer Res 68: 2391-2399, 2008.

30. Thomson S, Petti F, Sujka-Kwok I, Epstein D and Haley JD: Kinase switching in mesenchymal-like non-small cell lung cancer lines contributes to EGFR inhibitor resistance through pathway redundancy. Clin Exp Metastasis 25: 843-854, 2008. 